roc. Int. Conf. on Quark Lepton Nuclear Physics

QULEN' 97

May 20-23, 1997

Osaka, JAPAN

BNL -64602

CONF- $970526-1$

\title{
H Particle Searches at Brookhaven
}

Robert E. Chrien ${ }^{\text {* * }}$

DISTRIUTION OF THIS DOCUMENT IS UNA HITED

aPhysics Department, Brookhaven National Laboratory, Upton, NY 11973

Following the suggestion by R. L. Jaffe twenty years ago [1], researchers have been trying to establish the existence of a six-quark object, termed the " $\mathrm{H}$ " dibaryon, predicted by the phenomenological quark bag model. This object quickly became the focus of experimental searches at several locations, including the AGS [2]. This search still continues, with perhaps the most active program being carried out at the $2.0 \mathrm{GeV} / \mathrm{c}$ beam line at the BNL-AGS. The research was considerably enhanced by the writing of two notable papers at BNL by Aerts and Dover [3,4], which gave quantitative predictions for $\mathrm{H}$-production cross sections in two very different reaction mechanisms. One of these, the formation of cascade atomic deuterium to form the $H$ by fusion, had been first suggested by P. D. Barnes [5]. The $2.0 \mathrm{GeV} / \mathrm{c}$ line [6] at the AGS was specifically designed to provide an adequate kaon flux for double strangeness and charge exchange reactions. It has been used for two $H$ searches, E813 and E836, as well as for several $\Lambda \Lambda$ searches, E885 and E906. These four experiments are setting significant limits on H-production. The results of these experiments are discussed, and descriptions of related $B$ searches at Brookhaven are given.

\section{The Beginning}

Perhaps a stable dibaryon.... With this rather tentative title, R. L. Jaffe [1], twenty years ago, electrified the community of experimenters in particle and nuclear physics with his suggestion that it was possible to uncover evidence for QCD in interactions between hadrons at rather modest excitation energies. Jaffe pointed out that the same quark bag model which described the $\mathrm{S}$-wave baryons and mesons so well also predicted the existence of certain relatively light dibaryons; one of particular interest is the assembly of uuddss quarks in relative $S$-orbits, coupling to form the $J^{P}=0$ assembly popularly known as the $\mathrm{H}$-dibaryon or " $\mathrm{H}$ " particle.

The interest in this object stems from the insight it might offer on quark dynamics. While it is termed a "dibaryon", it is important to distinguish it from the deuteron, a true dibaryon in the sense of being a loosely bound state of distinct baryons. The $H$ must be considered a composite of quarks with highly similar wave functions.

With a mass Jaffe estimated to be between the $\Sigma$-nucleon and the $\Lambda \Lambda$ masses, and with a predominately $\Delta \mathrm{I}=3 / 2$ weak decay to a $\Sigma^{-}$-proton mode, this object might be quite

"Research supported by the U. S. Department of Energy. 
easy to detect. Later Donoghue and colleagues [7] suggested a likely lifetime of $10^{-8}$ sec. provided the mass was near $2200 \mathrm{MeV} / \mathrm{c}^{2}$

This suggestion stimulated two decades of intensive experimental activity. Urged on by the promise of a relatively simple search, a number of quick experiments were done, and later, as the difficulties became more apparent, the experiments became more elaborate and painstaking.

This review does not aspire to be a comprehensive summary of these many searches. Rather, it describes half-a-dozen experiments at the Brookhaven AGS, which arguably offers the best facilities for such a search. In that smaller class, this review focuses on experiments done at the $\mathrm{D} 6$ beam line, which offers the best external kaon beam available in the world.

The experiments to be described here are listed in Table 1. The proposal numbers and submission dates are also indicated. These proposals are maintained on file at BNL; copies are available on request. Of course many of them have been published, and references are provided where appropriate. It is, however, interesting and instructive to compare them against the later publication.

Table 1

A CHRONOLOGY OF AGS H-SEARCH PROPOSALS

\begin{tabular}{|c|c|c|}
\hline \multirow{3}{*}{ 2) } & E-703, "Search for Exotic Six-Quark States" & 10 Dec 76 \\
\hline & \multicolumn{2}{|l|}{ E-810, "A Search for Quark Matter..." } \\
\hline & "V $V_{0}$ Production with $14.5 \mathrm{GeV} / \mathrm{c}$ Silicon Beams" (Addendum) & $4 \operatorname{Jan} 85$ \\
\hline 3) & E-813, "Search for a Strangeness-2 Dibaryon"* & 1 Jan 85 \\
\hline 4) & E-830, "A Search for the H-Particle & \\
\hline & Using the ${ }^{3} \mathrm{He}\left(\mathrm{K}^{-}, \mathrm{K}^{+}\right) \mathrm{nH}$ Reaction" & $4 \mathrm{Jan} 86$ \\
\hline 5) & E-836, "Search for a Strangeness-2 Dibaryon & \\
\hline & Using a ${ }^{3} \mathrm{He}$ Target"* & 7 May 86 \\
\hline 6) & E-864, "Production of Rare Composite Objects in & \\
\hline & Relativistic Heavy-Ion Collisions" & 28 Sep 90 \\
\hline & E-888, "Search for the Weak Decay of an H Dibaryon" & $16 \mathrm{Jan} 92$ \\
\hline 8) & E-896, "Search for a Short-Lived $H_{0}$ Dibaryon" & Aug 93 \\
\hline
\end{tabular}

They span a range of production mechanisms and detection techniques, including associated production in E703, diffractive dissociation in E888, heavy ion interactions in E810, E864, and E896, and double strangeness exchange in E813 and E836. Each has its special attributes, but the emphasis here is on the double strangeness exchange reaction, (denoted by asterisks), which offers the most reliable predictive theoretical basis.

\section{E7̧03-Search of Exotic Six-Quark States}

This experiment, proposed by Fitch and Kycia, was performed at the AGS using a $5.0 \mathrm{GeV} / \mathrm{c}$ proton beam. The H was searched for in the double associated production reaction, $p+p \rightarrow K^{+}+K^{+} X$, in the Princeton double-arm spectrometer, by looking for the $\Sigma^{-} \mathrm{p}$ decay mode. A sensitivity of $1 \mathrm{nb}$ was expected, and a crude estimate of the 


\section{DISCLAMMER}

This report was prepared as an account of work sponsored by an agency of the United States Government. Neither the United States Government nor any agency thereof, nor any of their employees, makes any warranty, express or implied, or assumes any legal liability or responsibility for the accuracy, completeness, or usefulness of any information, apparatus, product, or process disclosed, or represents that its use would not infringe privately owned rights. Reference herein to any specific commercial product, process, or service by trade name, trademark, manufacturer, or otherwise does not necessarily constitute or imply its endorsement, recommendation, or favoring by the United States Government or any agency thereof. The views and opinions of authors expressed herein do not necessarily state or reflect those of the United States Government or any agency thereof. 


\section{DISCLAMMER}

Portions of this docoment may be illegible in electronic image products. Images are produced from the best available original document. 
production cross section was made, suggesting a level of $50 \mathrm{mb}$ at $5 \mathrm{GeV} / \mathrm{c}$.

In the event, the desired sensitivity was not achieved, and a upper limit of about $30 \mathrm{nb}$ was reported in the paper of Carroll et al. [2]. In subsequent theoretical treatments, it has been generally held that this experiment did not place significant limits on $\mathrm{H}$ production (see for example ref [3]).

\section{E-810-A Search for QGP and other New Phenomena Utilizing Heavy Ion Collisions at the AGS}

In this experiment, carried out as part of the fixed-target heavy ion program at the AGS, a search was made in centrally enriched events using a time-projection-chamber in the Multi-Particle Spectrometer (MPS) at the AGS. With $14.6 \mathrm{~A} \mathrm{GeV} / \mathrm{c}$ Si ions on a $\mathrm{Pb}$ target, candidate events were selected with a topology consistent with $H \rightarrow \Sigma^{-} p$ decay events. This was the first attempt at the AGS to search for the $H$ in strangeness-rich heavy ion collisions. Longacre et al [8] reported finding 33 candidate events on a background of 11 , from a sample of 5000 . Estimates of H-production from a coalescence model have been made by Baltz et al. [9]. They estimate a production rate of fifty times less than observed in E810-or less than one net event. As a perusal of ref. [8] will show, heavy ion interactions produce a high density of tracks in the chamber, leading to difficulties and ambiguities in track reconstruction.

This embarrassment of riches has led to speculation that an extraordinary production mechanism, like creation of a quark gluon plasma, may be involved.

An interesting variation on the theme of $\mathrm{E} 810$ is offered by E896, which is like E810 but has several improvements. Using gold on gold at $11.6 \mathrm{~A} \mathrm{GeV} / \mathrm{c}$, this experiment uses lowmass distributed drift chambers and a high-field (5T) superconducting magnet to sweep out the charged particle background and observe the $H$ decays free of the backgrounds which are seen in E810. A scintillation neutron hodoscope provides detection of the $\Sigma^{-}$ decay to $n \pi^{-}$. E896 promises good sensitivity for lifetimes as short as typical hyperon lifetimes. The first trial of E896 has just been completed in the 1997 AGS run and a full data taking run will be done in early 1998.

No results are available yet for the other relativistic heavy ion proposal on the list-E864. This experiment involves finding objects with anomalous charge/mass ratios, presumably indicating the presence of "strange matter" conglomerates.

\section{E888-Search for the Weak Decay of an $\mathbf{H}$ Dibaryon}

Using a neutral beam produced from 1.4 interaction length $\mathrm{Cu}$ target of the B5 beam line of the AGS, this experiment looked for $H$ dibaryons with two techniques: a) by observing $\Lambda$ decays in a 10 meter vacuum tank downstream of the target and which do not point back to the target, $b$ ) by placing a dissociator in the neutral beam and looking for $\Lambda$ pairs pointing to it. Initially ref. [12], using method a) claimed two candidate events, causing quite a stir. The claimed candidates were subsequently withdrawn. The published report of b) [13] identifies no candidates. Since these are decay measurements, they are sensitive to the $\mathrm{H}$ lifetime, which according to ref. [7] is of the order of $10 \mathrm{~ns}$ for a mass of $2.15 \mathrm{GeV} / \mathrm{c}^{2}$. Method a) is insensitive below $1 \mathrm{~ns}$, while b) is insensitive below 10 ns. 


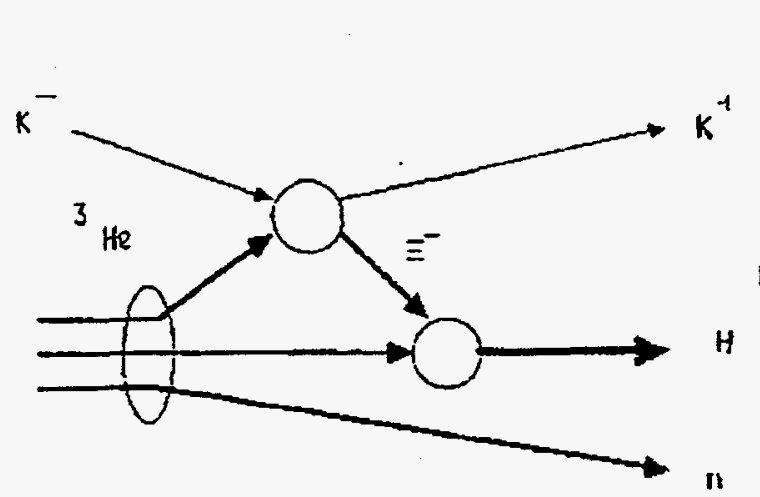

One Step Process

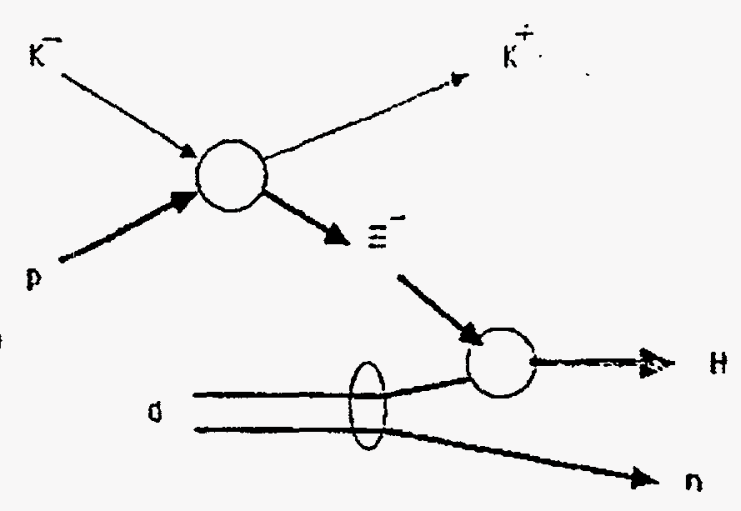

Two Step Process

Figure 1. Reaction Mechanisms for $\left(K^{-}, K^{+}\right)$

\section{Double-Strangeness Exchange Searches}

After Jaffe's original suggestion, it was quickly realized that the reaction easiest to calculate and offering optimal sensitivity was the double-strangeness and charge exchange reaction $\left(K^{-}, K^{+}\right)$. The direct measurement of $B$ production offers the virtue of independence of $H$ lifetime, and its creation in the simplest possible nuclear systems is the most amenable to theoretical model analysis. Two papers authored by Aerts and Dover $[3,4]$ calculated $H$ formation cross sections for both in-fight reactions, and for stopping $\Xi$ reactions. Schematic diagrams for these two reaction mechanisms are shown in Figure 1. The effect of the double-strangeness and charge exchange is to transform a pair of protons into an $\mathrm{H}$ plus a neutron.

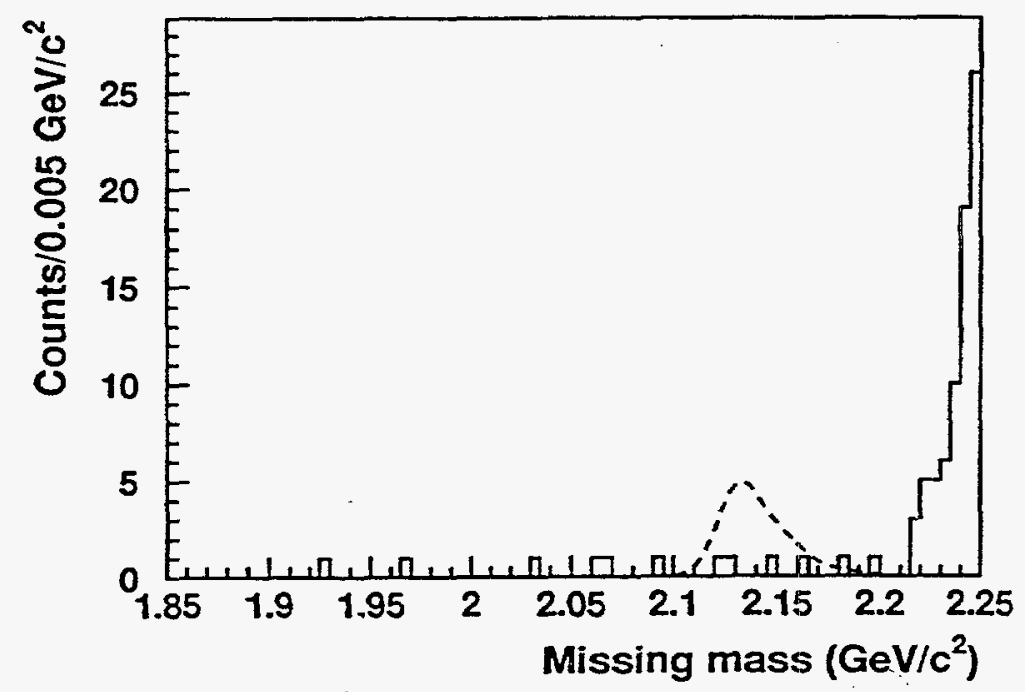

Figure 2. The expected peak from Aerts and Dover superimposed on the data of $\mathrm{E} 836$

In the case of in-flight production, Aerts and Dover calculated the cross sections for the 
nucleus ${ }^{3} \mathrm{He}$, the simplest possible system containing the two protons. Their calculation provided the basis for the E836 proposal of Table 1.

The calculation of $H$ formation from capture of the $\Xi^{-}$on deuterium was made in response to a suggestion of Peter Barnes in the Second LAMPF II Workshop Proceedings [5]. Barnes pointed out that the $H$ could be formed in the creation of a $\Xi^{-}$deuterium atomic system, whose subsequent fusion to the $\mathrm{H}$ would be signaled by the emission of a neutron. The neutron's kinetic energy would be a measure of $\mathrm{H}$-particle binding. Of course, since $\Xi^{-}$beams are not available, this process necessitates the placement of an out-of-beam target chamber adjacent to the $\Xi$ production vessel, as described in the discussion of E813. The geometry limitations imposed on the capture of the $\Xi^{- \text {'s }}$ in this method, and the unavoidable neutron background are serious difficulties, as we shall see below.

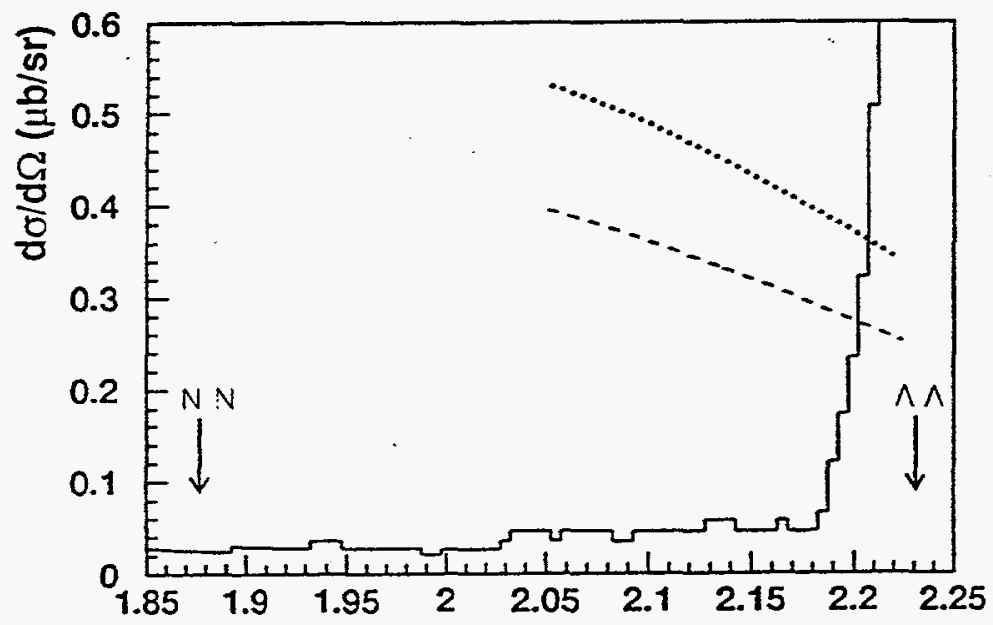

Figure 3. The cross section predicted by Aerts and Dover compared to the data. The lower of the two curves is a recalculation using more recent $\Xi$ cross sections.

Either of these methods requires a suitably intense $\mathrm{K}^{-}$beam, which was unavailable at the time E813 and E836 were proposed. To remedy this lack, the BNL group undertook studies of a $2 \mathrm{GeV} / \mathrm{c}$ beam line at the AGS. The design and construction of this line, funded jointly by the High Energy and Nuclear Physics programs of the USDOE, made those experiments possible. The line provides an intensity of $2.0 \times 10^{6} \mathrm{kaons}$ at $1.8 \mathrm{GeV} / \mathrm{c}$, and a purity of $1: 1$ or better, for kaons to pions. This purity was made possible by two stages of separation, using novel beam separators used in the successful $2.0 \mathrm{GeV} / \mathrm{c}$ line of KEK. The design of this line was due to Phil Pile of the BNL Medium Energy group, and the construction was completed in 1990.

The experiments at the D6 line use a large open (non-focusing) spectrometer to provide the $\mathrm{K}^{+}$analysis. This magnet has been used in all D6 line experiments to date.

\subsection{E836-Search for a Strangeness -2 Dibaryon Using a ${ }^{3}$ He Target}

Although not the first H-search experiment using the $2.0 \mathrm{GeV} / \mathrm{c}$ beamline, this was the first to be completed [14]. As explained in ref [4] a simple missing mass experiment, 
without requiring detection of the spectator neutron, suffices to display the expected dibaryon, even though the expected peak would be Fermi-broadened by motion of the diproton pair in ${ }^{3} \mathrm{He}$. The data for E836 were taken in the 1994 AGS run.

The results of this search are contained in a publication which appeared just at the time of this conference [14]. The results can be summarized in Figures 2 and 3 . Only a few remarks need be added here.

The results rule out $\mathrm{H}$-particle production in the range below $2200 \mathrm{MeV} / \mathrm{c}^{2}$. The range below the mass 1875 was already ruled out by other experiments [10]. Because of the Fermi-broadened peak expected, and because of the onset of quasi-free $\Xi$ production, this experiment is not sensitive to the interesting range between the $\Lambda \Lambda$ mass (2230) and 2200 $\mathrm{MeV} / \mathrm{c}^{2}$.

It should be acknowledged that similar upper limits have been placed in an experiment reported by Ahn et al.[11] on a scintillating fiber target in KEK experiment E224. This experiment requires an estimate of the "effective" number of S-wave diproton pairs in a carbon nucleus, so that only an approximate comparison to the Aerts and Dover calculation can be made.

LD2 Vessel
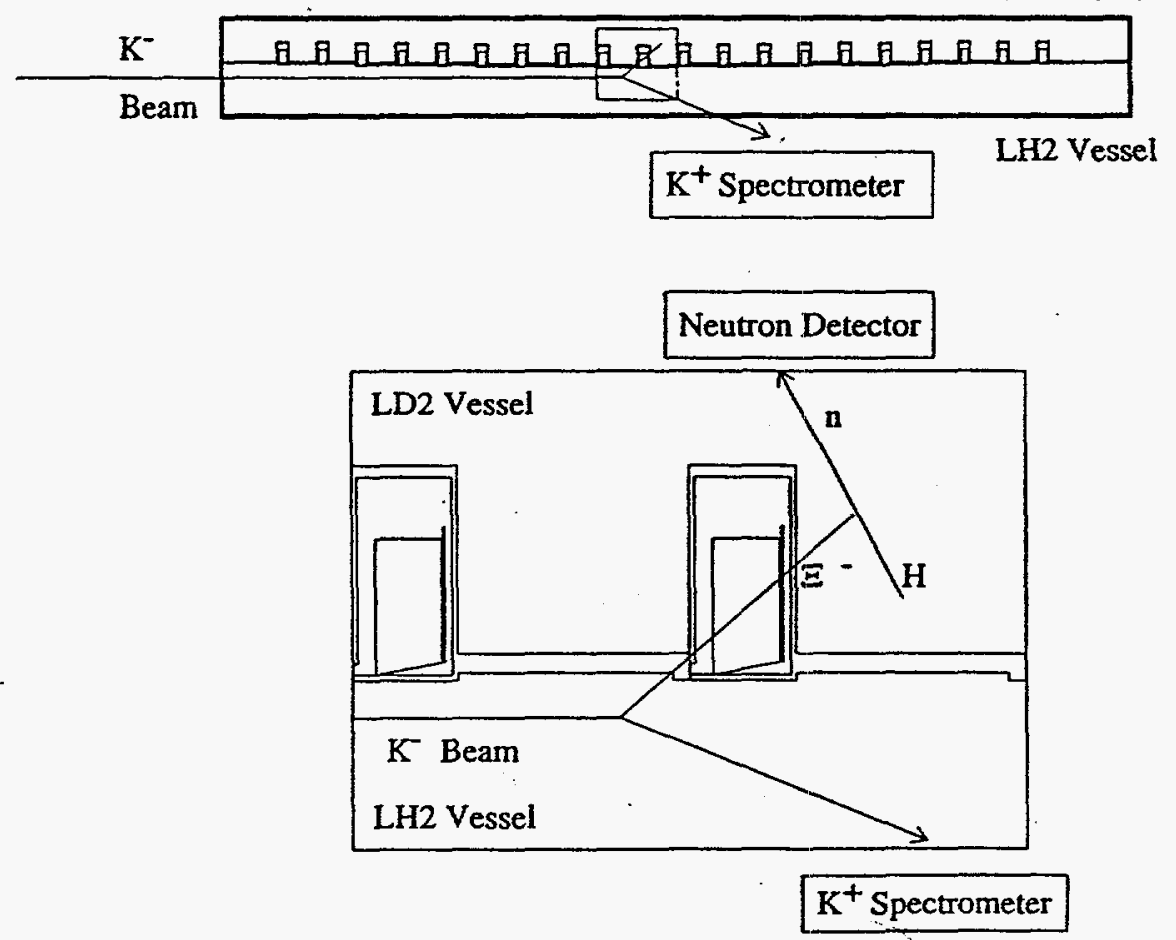

Figure 4. Target design for implementing the $\mathrm{H}$ search using stopping $\Xi$ 's. 


\subsection{E813-Search for a Strangeness -2 Dibaryon}

This experiment was done in several sections, in the years 1992, 1993, and 1995. As previously described, this experiment was accomplished with a design featuring a 60 cm-long cryogenic target partitioned into two halves; one containing hydrogen through which a $K^{-}$beam was introduced, the second containing deuterium to capture the $\Xi$ produced in the other. This design is meant to effect the sequence of two-body reactions of Figure 1. It is shown in Figure 4. The partition is covered with tungsten degrader plates on which are placed silicon detector strips. The passage of a suitably slow $\Xi$ produced a signal depositing $1.5 \mathrm{MeV}$ in the Si detectors which identified a $\Xi$ likely to stop in the deuterium target. This tag is used to identify neutrons detected by a large array of plastic scintillation detectors surrounding the target.

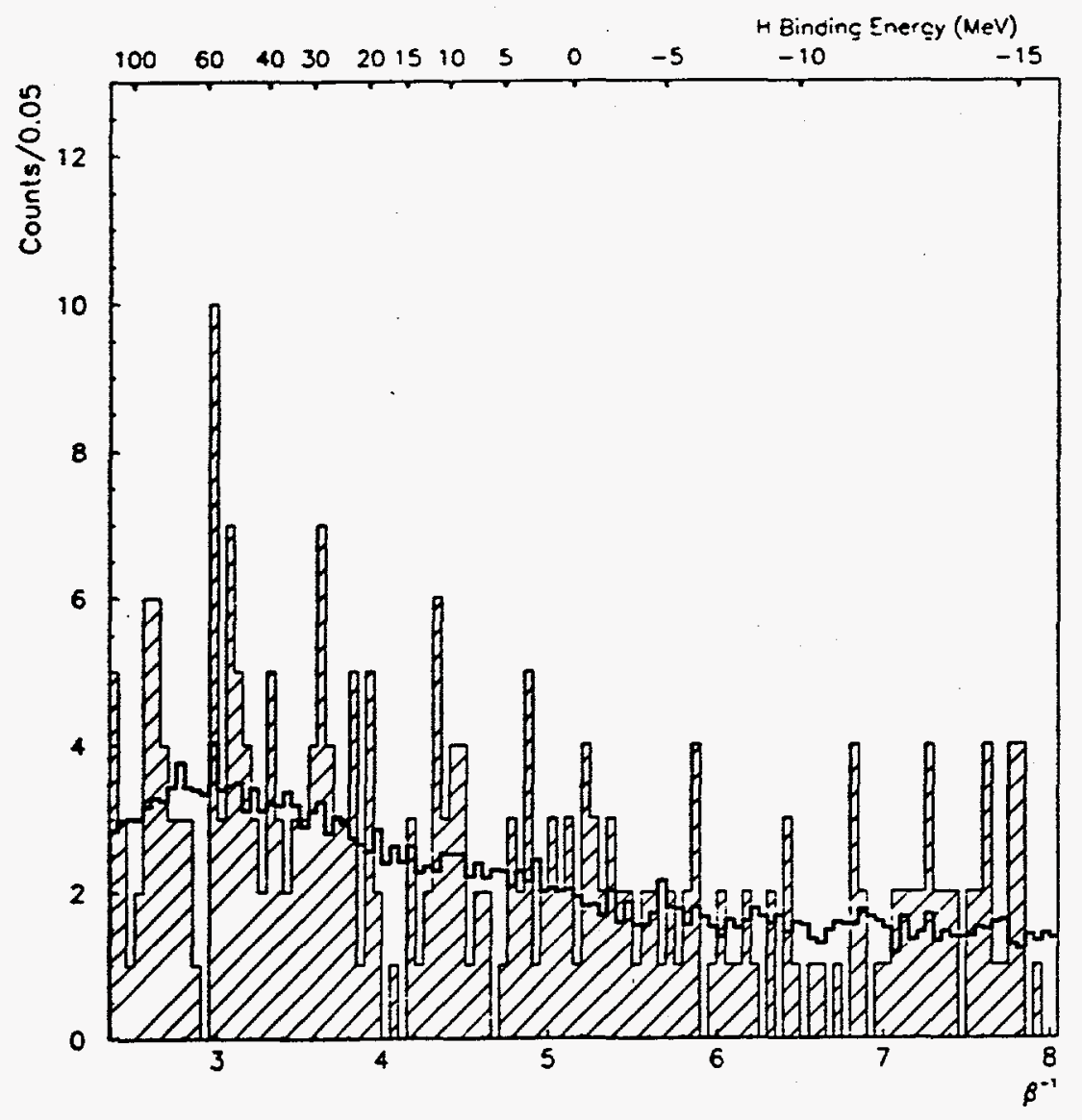

Figure 5. Neutron spectrum from the 1993 data of E813.

The geometry of the partitioned target makes the $\Xi^{+}$capture process inefficient, and difficult to calculate. Further it creates an unavoidable neutron background. In the implementation of this experiment, it was found that the original estimate for the neutron signal was over two orders-of-magnitude too high. However, the reduced signal will still 
lead to significant limits in the important region just below the $\Lambda \Lambda$ mass.

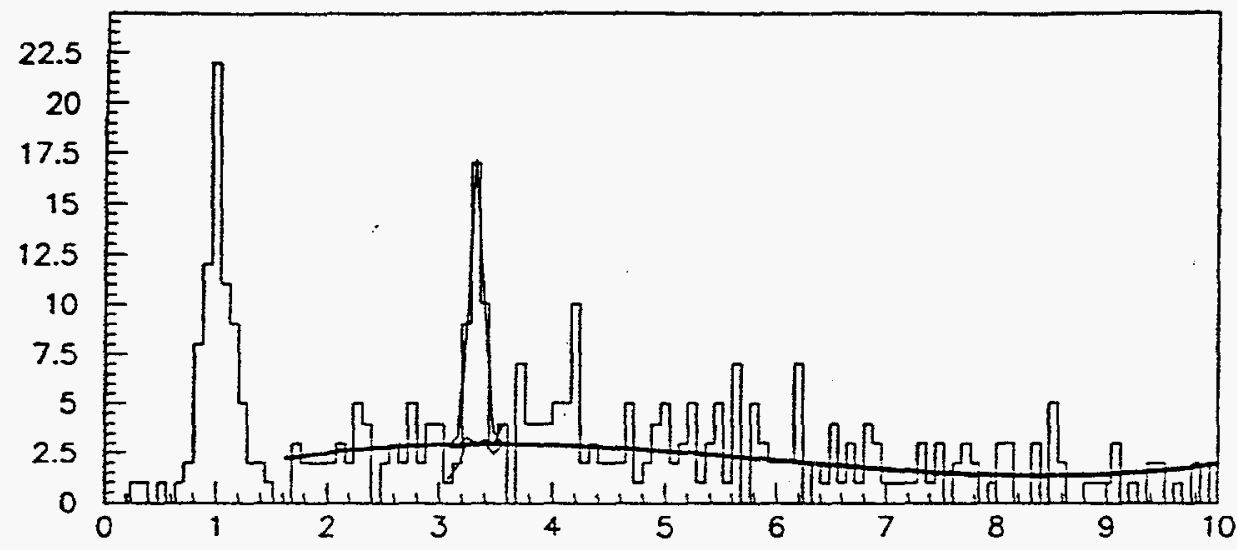

Figure 6. The neutron peak observed by the fusion of $\Sigma^{-}$hyperons with protons in the dual vessel cryotarget of E813

The neutron signal depends strongly on the branching ratio for $\mathrm{H}$ formation and that ratio was estimated by Aerts and Dover [3] to range from 0.8 at zero binding to 0.1 at a binding energy of $100 \mathrm{MeV}$. Well over $10^{12} \mathrm{~K}^{-}$were incident on target, in three installments. About $2 / 3$ of the data set has been analyzed to date. The neutron data from the 1993 run is displayed in Figure 5, which comprises about $1 / 2$ of the total. From an irradiation of $7.810^{11} \mathrm{~K}^{-}$, it is estimated that about 100 tagged stopping $\Xi$ 's were produced. From the surrounding neutron detectors, which had a solid angle efficiency product of about $10 \%$, one can estimate that only eight $H$-signal neutrons are produced. Obviously it is difficult to set significant limits for $\mathrm{H}$ production from these data, especially for regions of binding energy greater than a few tens of $\mathrm{MeV}$.

Since this mass range - that is below $2200 \mathrm{MeV} / \mathrm{c}^{2}$ - is already ruled out by E836, this lack of sensitivity is not crucial for E813. It is anticipated that the complete set will allow a significant upper limit -that is above $90 \%$-to be set in the important range from 0 to $30 \mathrm{MeV}$ of $\mathrm{H}$ binding.

While the verdict on E813 is still out, it is important to take note of a calibration experiment which was performed in 1995 to validate the sensitivity of E813 [16,17] This was a study of the reaction $\left(\pi^{-}, K^{+}\right)$, which produces a $\Sigma^{-}$and makes a $\Sigma$-deuteron atomic system. The known cross section [18] of $\Sigma$ production allows us to calibrate the efficiency of the two-target system. The neutron released in the $\Sigma$-proton fusion is detected at a level well above background as shown in Figure 6 . This calibration is essential in demonstrating success in tagging the stopping hyperon and detecting the resulting monoenergetic neutron. 


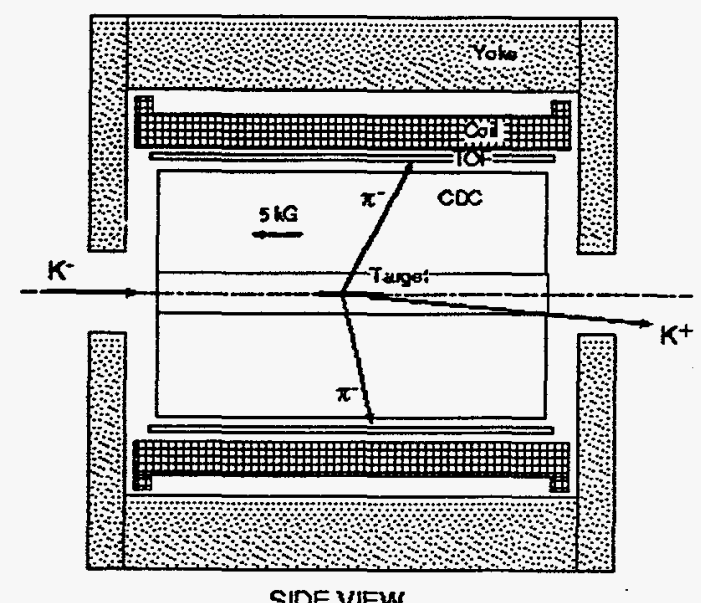

SIDE VIEW

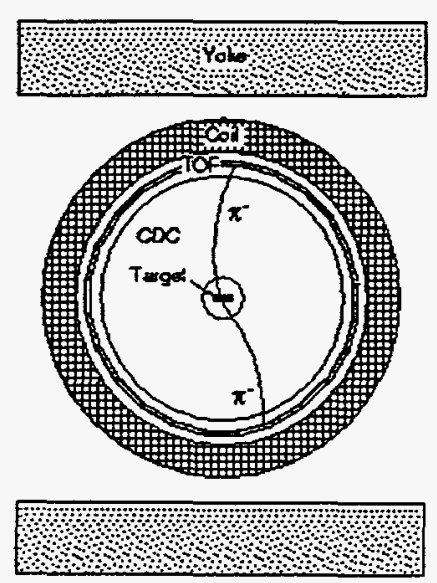

FRONT VIEW

Figure 7. A schematic view of the solenoidal detector CDS and how it detects the sequential decay pions from $\Lambda \Lambda$ hypernuclei.

\section{Other Experiments-E885 and E906}

Establishing the existence of Lambda-lambda hypernuclei places limits on the masses of a hypothetical H-dibaryon. The Lambdas could not coexist in the nuclear medium, since they would decay strongly to an $\mathrm{H}$.

Several experiments at the AGS D6 beamline have been carried subsequent to E813 and E836 which have a bearing on the existence of $H$ dibaryons bound in a nuclear medium. In E885, a search for doubly-strange hypernuclei was carried out with a scintillating fiber detectors viewing a diamond target. Using the neutron detectors of E813, a peak corresponding to the neutron de-excitation of a $\Lambda \Lambda$ bound state is being searched for in association with weak decay pions picked up in the sci-fi stacks.

A search for $\Lambda \Lambda$ hypernuclei is being done with the CDS- the Cylindrical Detector system constructed at the INS, Tokyo. The CDS features a $0.5 \mathrm{~T}$ solenoidal magnet, the interior of which contains coaxial cylindrical chambers. As diagrammatically shown in Figure 7, the CDS will track the pair of sequential weak decay pions which signal the two decrements of strangeness marking the transition of the doubly strange $\Lambda \Lambda$ hypernucleus back to ordinary matter.

The CDS includes a cylindrical drift chamber system with 550 channels in twelve layers of axial and stereo layers in a cylinder $30 \mathrm{~cm}$ by $92 \mathrm{~cm}$. in length. A 640 channel proportional counter surrounds the drift chamber to provide precise position along the beam line. A 22-element scintillation hodoscope encloses these packages for precise event timing. The determination of an coincidence between the decay pions and a $\left(\mathrm{K}^{-}, \mathrm{K}^{+}\right)$trigger, together with a precise measurement of the decay pion momenta, provides identification of a $\Lambda \Lambda$ event. The dilambda could reside in a bound state, or in a hyperfragment from the reaction.

The CDS avoids the necessity of dealing with stopping $\Xi$ 's, where the $\Xi$ is produced 
by a quasifree process and stopped in a target, where it forms an atomic system. This method is inefficient because because most $\Xi$ 's decay before stopping. If the secondary interaction of the $\Xi$ inside the nuclear medium occurs, there is a reasonable probability of forming a hyperfragment containing two $\Lambda$ 's. As discussed in the E906 proposal [19], the rate of formation for ${ }_{\Lambda \Lambda}^{3} \mathrm{H}$, for example, is estimated to be $0.27 \%$ in the quasifree part of the $\left(\mathrm{K}^{-}, \mathrm{K}^{+}\right)$reaction on a beryllium target. This is considerably larger than would be achievable in the stopped $\Xi$ method.

The first operation of the CDS occurred in the 1997 AGS run just completed. The successful operation of the system was demonstrated, and interesting candidate events are now being examined.

\section{Conclusions}

A number of $H$ searches have been carried out at the BNL-AGS using a variety of approaches. These have so far produced no unequivocal evidence for these objects in the mass range originally suggested by Jaffe. Several of them, however, have produced upper limits which rule out $H$ production as calculated by conventional theories such as the various coalescence models. Several searches continue by exploring exotic mechanisms like the creation of quark-gluon plasmas. It seems likely that new theoretical approaches are needed to understand the data now in hand or soon to be produced.

\section{REFERENCES}

1. R. L. Jaffe, Phys. Rev. Lett. 38(1977)195.

2. A. S. Carroll et al., Phys. Rev. Lett. 41(1978)777.

3. A. T. M. Aerts and C. B. Dover, Phys. Rev. D28(1983)450.

4. A. T. M. Aerts and C. B. Dover, Phys. Rev. D29(1984)433.

5. P. D. Barnes, Los Alamos Report LA-972-C, Vol.1 (1982)315.

6. P. H. Pile et al., Nucl. Instr. Meth. A321(1992)48.

7. J. F. Donoghue, E. Golowich, and B. R. Holstein, Phys. Rev. D34(1986) 3434.

8. R. Longacre, Nucl. Phys A590(1995)472c.

9. A. J. Baltz et al., Phys. Lett. B3265(1994) 7.

10. H. Ejiri et al. Phys. Lett. B228(1989) 62.

11. J. K. Ahn et al. Phys. Lett. B378(1996) 53.

12. J. Belz et al. Phys. Rev. Lett. 76(1996) 3277; and addendum, to be published in Phys. Rev. C (1997)

13. J. Belz et al. Phys. Rev. D53(1996)R3487

14. R. W. Stotzer et al. Phys. Rev. Lett. 78(1997)3646

15. T. Lijima et al. Nucl. Phys. A546(1992)588

16. M. Burger, Dissertation, Albert-Ludwigs-Universitat Freiburg, (1996)

17. L. Gan, Private Communication, University of Manitoba (1997)

18. P. Livanos, Thesis University of Paris-Sud, Orsay (1980)

19. T. Fukuda, E906 Proposal and Private Communication 